\section{Esophageal involvement by pemphigus vulgaris resulting in dysphagia}

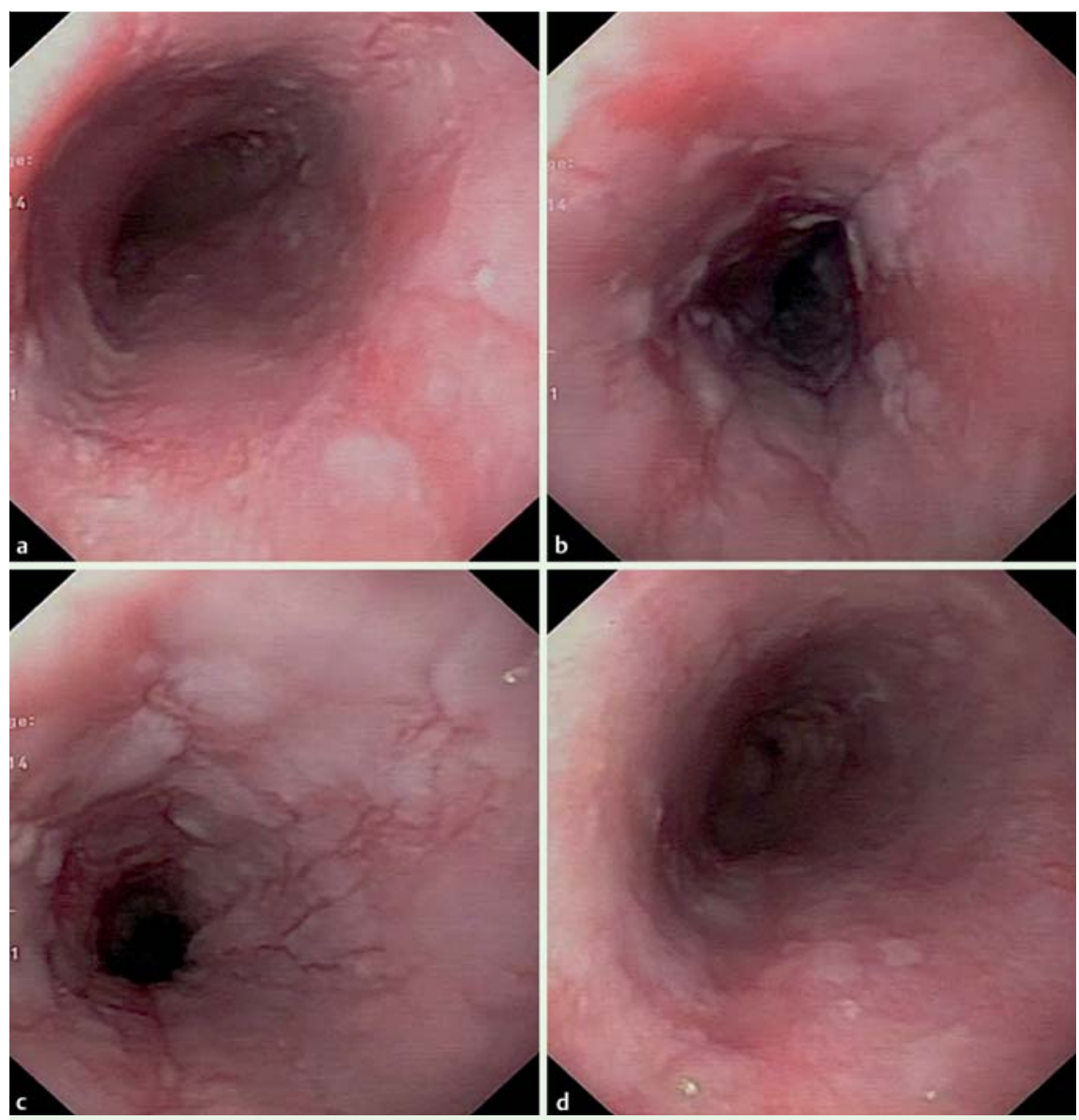

Fig. 1 In a 44-year-old patient with dysphagia because of pemphigus vulgaris that involved the esophagus, endoscopic esophageal views show mucosal exfoliation and confluent linear erosions that become less severe distally: $\mathbf{a}, \mathbf{b}$ upper esophagus; $\mathbf{c}$ mid esophagus; $\mathbf{d}$ lower esophagus.

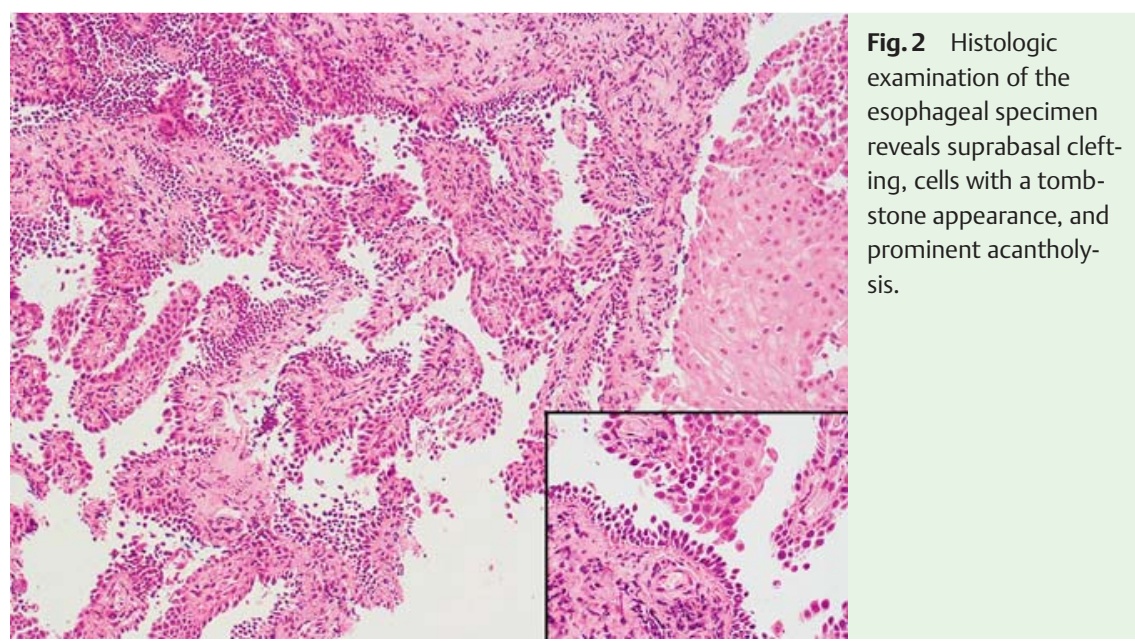

A 44-year-old man was admitted to our institution because of the appearance of painful cutaneous, oral, and genital vesicles. His previous medical history was unremarkable. He had had pharyngitis treated with amoxicillin 1 month before the hospitalization. On physical examination, symmetrically erythematous plaques, covered with vesicles, were seen. Because of subsequent dysphagia for solid food, he underwent esophagogastroduodenoscopy.

The endoscopic examination revealed esophageal mucosal exfoliation with multiple linear and confluent furrows in the upper and middle esophagus ( $\bullet$ Fig. 1 ) that were less severe in the lower esophagus. The gastric and duodenal mucosa was preserved, without mucosal breakage. Biopsy specimens were taken from lesions in the upper and middle esophagus, with stripping of the mucosa on withdrawal of the biopsy forceps ( Video 1 ). The histologic examination showed suprabasal clefting, cells with a tombstone appearance, and prominent acantholysis, suggestive of pemphigus vulgaris ( Fig.2). At the same time, a skin lesion biopsy was performed, which confirmed the diagnosis ( $\bullet$ Fig. 3 ).

Systemic corticosteroid treatment was started but was unsuccessful; during subsequent immunotherapy and the intravenous administration of monoclonal antibodies, the symptoms and signs decreased.

Pemphigus vulgaris is a rare autoimmune disease in which bullae of the skin and oral mucosa form as a result of acantholysis mediated by circulating immunoglobulin $\mathrm{G}$ autoantibodies against intercellular antigens of stratified epithelia. Pemphigus vulgaris can involve other mucosal surfaces that have squamous epithelium, such as those of the esophagus, nasopharynx, conjunctivae, cervix, and anus [1]. The prevalence of esophageal involvement in autoimmune bullous skin disease is $67.8 \%$ [2], but esophageal involvement may be underrecognized or misdiagnosed without a proper endoscopic evaluation

\section{Video 1}

Biopsy specimens are taken from lesions in the upper and middle esophagus, with stripping of the mucosa on withdrawal of the biopsy forceps. 


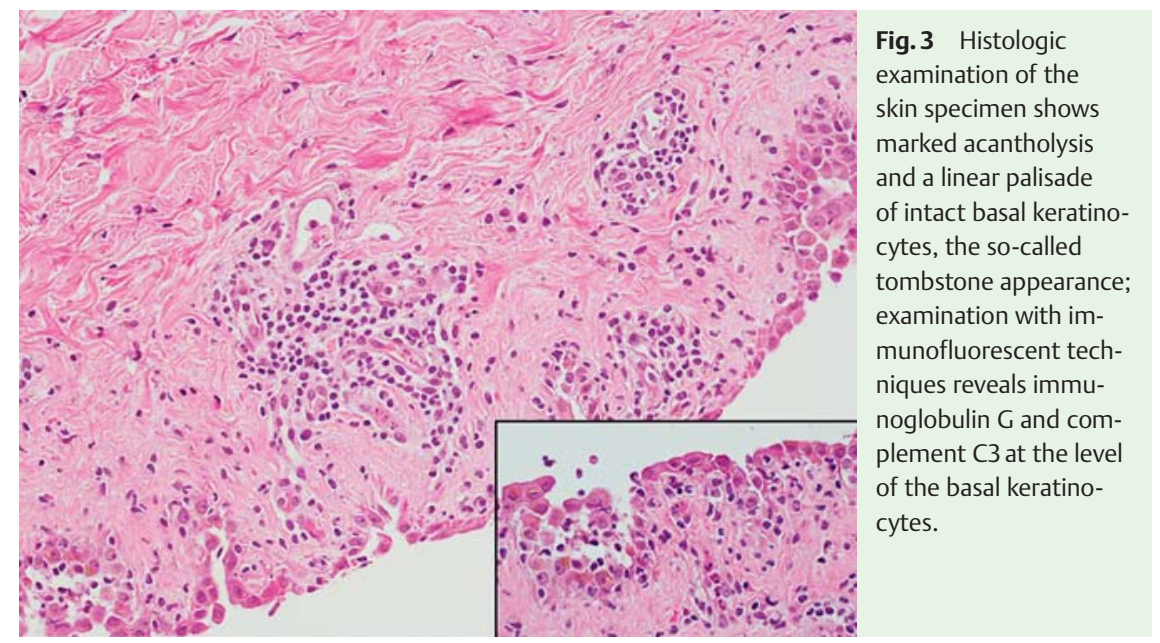

[3]. Commonly, it is characterized by vesicles, superficial erosions, red and erythematous longitudinal lines, a circumferential crack with peeling, and a whitish mucosa with extensive bleeding along the entire organ. Pemphigus vulgaris may also be induced by drugs, including d-penicillamine and angiotensin-converting enzyme inhibitors $[4,5]$. Corticosteroids and immunosuppressive agents are considered the first-line treatment. If untreated, pemphigus vulgaris can be fatal.

Endoscopy_UCTN_Code_CCL_1AB_2AC_3AH

\section{Competing interests: None}

\section{References}

1 Bystryn JC, Rudolph JL. Pemphigus. Lancet 2005; 366: $61-73$

2 Galloro G, Mignogna M, de Werra C et al. The role of upper endoscopy in identifying oesophageal involvement in patients with oral pemphigus vulgaris. Dig Liv Dis 2005; 37: 195-199

3 Hokama A, Yamamoto Y, Taira Ket al. Esophagitis dissecans superficialis and autoimmune bullous dermatoses: a review. World J Gastrointest Endosc 2010; 2: 252 - 256

4 Duhra P, Foulds I. Penicillin-induced pemphigus vulgaris. Br J Dermatol 1988; 118: 307

5 Ruocco V, Satriano R, Guerrera V. "Two step" pemphigus induction by ACE-inhibitors. Int J Dermatol 1992; 31: 33-36

\section{Bibliography}

Dol http://dx.doi.org/

10.1055/s-0034-1391905

Endoscopy 2015; 47: E271-E272

(c) Georg Thieme Verlag KG

Stuttgart · New York

ISSN 0013-726X

${ }^{1}$ Unit of Gastroenterology and Digestive Endoscopy, Arcispedale Santa Maria Nuova-IRCCS, Reggio Emilia, Italy

${ }^{2}$ Unit of Digestive Endoscopy, Ospedale Cesare Magati, Reggio Emilia, Italy

${ }^{3}$ Unit of Pathology, Arcispedale Santa Maria Nuova-IRCCS, Reggio Emilia, Italy ${ }^{4}$ Center of Hemostasis and Thrombosis, Arcispedale Santa Maria Nuova-IRCCS, Reggio Emilia, Italy 